Wakefield J. and Kassem M. (2020). "Improving site access verification and operator safety in smart and sustainable assets: a pilot study in a UK decarbonisation project" In: Proc. 37th CIB W78 Information Technology for Construction Conference (CIB W78), São Paulo, Brazil, pp. 374-384. DOI: http://dx.doi.org/10.46421/2706-6568.37.2020.paper027

\title{
IMPROVING SITE ACCESS VERIFICATION AND OPERATOR SAFETY IN SMART AND SUSTAINABLE ASSETS: A PILOT STUDY IN A UK DECARBONISATION PROJECT
}

\author{
James Wakefield $^{1}$, and Mohamad Kassem ${ }^{2}$
}

\begin{abstract}
The built environment is increasingly shifting towards smart and sustainable cities. This transformation is accompanied by the implementation of new digital and smart technologies, and their supporting infrastructure. This advancement poses new challenges to the process of operating the built environment and in particular, to the management of new risks associated with use and operation of a more complex built environment. There is still a dearth of studies proposing new ways to cope with risks from the increased sophistication in the built environment.

This paper analyses the ability of an emerging technology and process for site access verification. The aim is to improve control measures in respect of the operational risk profile. HyDeploy, a UK based sustainable built environment gas decarbonisation pilot project, is used as a study case. HyDeploy is a project aiming to demonstrate that using hydrogen blended gas for heating and cooking is just as safe and convenient as the gas used currently in the UK.

The paper presents the current process for site access verification used in the project, before investigating the potential benefits of the application of an emerging technology for site access procedure. The paper concludes that the emerging procedure has the potential to improve both the compliance position of site owners, and the overall operator safety on site.
\end{abstract}

Keywords: Smart Cities, Compliance, Asset Lifecycle Operation, Health and Safety, Site Access Verification, Individualised Training Experiences

\section{INTRODUCTION}

The emerging challenges associated with growth in population and urbanisation are in part addressed by solutions for the digitalisation of the urban environment (Heaton and Parlikad 2019). Rapid urbanisation trends in the period up to 2027 requires investments up to $\$ 78$ trillion in global infrastructure (PricewaterhouseCoopers, 2017). Global warming is a key area of concerns where digitalisation of the urban environments is part of an effort to decarbonise energy consumption. Tools that can predict potential faults with infrastructure, to intelligent design of built environments that can filter air pollutants, technology has a promising role in improving built environments (Ahmed et al., 2017). Many cities are increasingly referred to as smart cities, with the city being

1 Associate Professor, Mechanical and Construction Engineering, Northumbria University Newcastle, Newcastle upon Tyne, UK, mohamad.kassem@northumbria.ac.uk

$2 \mathrm{PhD}$ Researcher, Northumbria University Newcastle, Newcasle upon Tyne, UK, jwakefield@environmentalessentials.co.uk 
"smart" meaning that "actors as multiple stakeholders (or societal initiatives guiding these actors) aim for sustainable solutions adopting digitalisation and other technological advancements" (Öberg, Graham and Hennelly 2017, P. 470).

Regardless of the drivers for the digitalisation of the urban environment - be they sociological, economical or ecological - hardware that supports increasingly digitised urban environments will be required to be safely and efficiently maintained according to the regulatory frameworks and the business models of the day. In such a context, this paper examines a specific use case about access verification and operator safety in a decarbonisation project using hydrogen blended gas for heating and cooking.

\section{LITERATURE REVIEW}

Workforce health and safety $(H \& S)$ in the construction sector (that being the sector responsible for delivering the future built environment) is a global concern. The construction sector has one of the highest fatal injury rates in both the developed and the developing world (Kassem et al., 2017). Efforts to improve the H\&S performance of the construction sector are plentiful and until recently studies have largely focused on H\&S issues on the construction site. However, facilities management and in particular the operation and maintenance of the existing built environment has a much higher rate of injury and illness when compared to all other fields of employment (Wetzel and Thabet, 2018). Tools and workflow enabled by Building Information Modelling (BIM) have the potential to support the transfer of hazard mitigation and safety data to operatives involved in assets operation and maintenance (Wetzel and Thabet, 2018). Carbonari, Stravoravdis \& Gausden (2018) assert that building information models can be exploited to support the delivery of more efficient facilities management services. Despite the recognised benefits of BIM in supporting workforce $\mathrm{H} \& \mathrm{~S}$, the current adoption of BIM in the operational phase of asset is minimal in comparison to the design and construction phases (Hilal, Maqsood \& Abdekhodaee, 2019).

$\mathrm{H} \& \mathrm{~S}$ training is key to improve the safety of workforce at all stages of an asset life cycle. There are many opportunities to exploit the potential of e-learning and associated digital learning technologies as a mechanism for delivery of hazard related information and training. Construction safety training delivered via an e-learning mode resulted in increased safety standards when compared to construction safety training delivered in the traditional space (Ho and Dzeng, 2010). The overall performance of computer-aided technologies to deliver construction site safety training is superior in several technical aspects compared to traditional tools, specifically, representing actual workplace situations, providing text-free inter-faces, and eliciting better user engagement (Gao, Gonzalez and Yiu, 2019). Other recognisable benefits of computer aided technologies specifically for construction include the ability of e-learning to support effective health and safety training delivery in a range of languages (Williams et al., 2010). According to Blocker (2005), e-Learning has the potential to address operational issues such as reducing costs, providing greater access to information and accountability for learning, and increasing employee competence and competitive agility. There is however much debate about factors affecting quality of e-learning outcomes. Ertmer \& Newby (2013) assert that many instructional designers are operating with limited pedagogical knowledge and designers must understand the position of the practitioner in order to be able to accurately design effective materials.

Highly engaging types of safety training can have a positive impact on safety performance (Shamsudin, 2018). To be effective the training must include content that is 
Improving site access verification and operator safety in smart and sustainable assets: a pilot study in a UK decarbonisation project

relevant to the trainee lives (Aik and Tway, 2006). Such understanding also informs efforts to leverage technology to provide short relevant training content at a time when it is most relevant to the task in hand, so called micro credentials (or credentialing). Such a training approach offers the opportunity to disrupt the time-based education and training model and recognises the competence required for tasks that are directly related to employment (Harvey, 2018). One such example of the application of micro credential training in the facilities management domain in presented in this paper. In this application, specific competencies of contractor employees are leveraged as credentials for site access in the HyDeploy project at Keele University in the UK.

\section{CASE STUDY}

HyDeploy is a UK energy decarbonisation demonstrator project which is designed to demonstrate for the first time that a blend of hydrogen and natural gas can be distributed and utilised safely and efficiently in the UK distribution network without disruptive changes for consumers.

The 10 month project involves blending up to $20 \%$ hydrogen into the Keele University gas network, delivering low carbon heat to customers without requiring disruptive and expensive changes in their homes. In addition to demonstrating the feasibility of the specific technology the project enables key stakeholders including the UK Government, public safety experts, the energy industry and academia to develop and understand the science base, the regulatory position, the supply chain and customer perceptions of hydrogen as a decarbonising element of the energy system. These insights are key to understand the wider process of feasibility testing and subsequently the adoption of energy decarbonising projects of all types.

Under the UK Climate Change Act, the UK must reduce its carbon emissions to net zero by 2050. Over a third of UK carbon emissions are from heat, with little progress having been made in decarbonising this sector to date. Over 23 million UK households (83\%) are connected to a gas supply and with gas being a flexible and convenient way to deliver heat, a key challenge is to reduce gas carbon content. Unlike natural gas, when hydrogen is burned it does not produce carbon dioxide. The hydrogen used for the project is produced by an onsite electrolyser (supplied by renewable electricity) which splits water into oxygen and hydrogen before blending the hydrogen into the Keele University gas network. This means that in general terms a blend including 15\% hydrogen would have a $15 \%$ decarbonising effect.

Keele University was selected as the home for HyDeploy and a number of other smart energy research projects on account of the Keele University campus being representative of a small town; this is in terms of its population demographics, its land mass, and land usage. The campus incorporates 101 residential buildings, 8 multiresidential buildings, 17 extensive office blocks and laboratories and 7 recreational and service facilities. Keele University owns the utilities infrastructure (including the gas network) and as such is a licenced transporter and carrier of gas. Keele University campus then is an ideal provider of a "living laboratory" for smart energy projects such as this one.

UK Gas Safety (Management) Regulations (GS(M)R) govern gas transportation and supply in the UK. In order that a hydrogen blend gas can be transported across and within any part of the UK gas grid, a specific exemption to the GS(M)R is required, this can only be granted by the UK Health and Safety Executive (HSE). Exemptions are permissible only if the health and safety of persons likely to be affected will not be 
prejudiced by the change process, with the change process on this case being the introduction of the hydrogen blend to the grid.

Permission for the pilot was granted by the UK HSE on the basis of extensive pre pilot project research and testing by the UK Health and Safety Laboratory (HSL) in partnership with Keele University and a number of commercial partners (including the UKs biggest gas distributor Cadent) which demonstrated that a $20 \%$ blend of hydrogen by volume is as safe as natural gas. The scope for the pre pilot project research and testing included (but not exclusively); network materials assessments, appliance and installations, [blended] gas characteristics and detection, and emergencies and maintenance procedures.

Due to innovative nature of this project and the likely H\&S risks to be involved, a number of specific site access verification arrangements (SAV) were specified for personnel (owner employed and contractor) responsible for the operation and maintenance of the network for the entire duration of the project. These HyDeploy specific SAV arrangements were implemented as part of a two-tier approach with Tier 1 being the existing general SAV control arrangements for the estate (tier one), and tier two being the HyDeploy specific SAV arrangements. The scope for this paper covers both tiers of access but is principally concerned with tier two, SAV arrangements, specifically for gas network engineers of first line contractors responsible for attending gas emergencies and carrying out meter works.

\section{RESEARCH METHODS}

A three stage qualitative content analysis approach was adopted to analyse data obtained from two research participants, identified via a purposeful sampling method. The participants represented the expert position on the estate owner side (Principal Mechanical Engineer for Keele University) and the contractor side (Future Networks Manager for Cadent Gas Ltd) respectively. Both participants have been extensively involved in the HyDeploy project since inception and as key members of the consortium stakeholder group have been instrumental in the development and implementation of the existing HyDeploy SAV control process.

It was identified that first hand insight would be required; this stage then was designed to ensure that the existing SAV arrangements reported by the researcher were an accurate representation of the SAV protocols which have been adopted for the project. Both participants took part in a semi structured interview process, Figure 1: Existing HyDeploy Cadent Operative Specific SAV Control Criteria was outputted as a result of this stage. Following an initial feedback opportunity both participants agreed that Figure 1 is an accurate representation of the SAV protocols which have been adopted for the contractor group.

Subsequent to formalising the Existing HyDeploy Cadent Operative Specific SAV Control Criteria flow, both participants completed an open-ended questionnaire which was designed to establish the degree of confidence on the part of the estates and the contractor representatives in the agreed Existing HyDeploy Cadent Operative Specific SAV Control Arrangement. The questionnaire also invited the research participants to identify and comment upon perceived opportunities to develop the SAV Control Arrangements for the contractor group. This component is undertaken with the benefit of two months prior live operation of the HyDeploy project and associated systems and procedures. 
Prior to completion of stage three open-ended questionnaire, the researcher shared Figure 2: Proposed Site Access Verification (SAV) Control Criteria Flow with both research participants. Figure 2 along with supporting information demonstrates a representative workflow that can be enabled with the application of new technology that can improve the H\&S of operatives and the H\&S compliance of the main contractor. Supporting information assisted the research participants in assessing and commenting upon that which is being presented. The general principle underpinning the proposed technology is the ability to provide operatives web-enabled access to information that is pertinent to the job being undertaken at any given time. This process can be linked to a range of required outcomes, such as the cultural proposition (public messaging), the efficiency position (how to do this job most efficiently), but the main focus of this study in on Health and Safety Compliance (risk mitigation).

\section{RESEARCH FINDINGS}

As the network maintenance supplier to the Keele University HyDeploy gas network Cadent are contractually obliged to ensure that a team of compliant service engineers is available 24 hours per day, 7 days per week, 365 days per year to attend to gas emergencies and meter works on the estate. Cadent determined an operational requirement that a team of 100 engineers would therefore need to be trained to meet HyDeploy Cadent Operative Specific SAV Control Criteria (tier two) in order to ensure compliance for access. The compliant status of the operative is then recorded back to the Cadent job scheduling system to ensure that only compliant operatives are available to be scheduled for works at the Keele University site. This represents a change in operational process for Cadent in that operative allocation to a job is ordinarily governed by job role qualification and the regional allocation of the operator. It is now the case that job allocation will controlled by the additional HyDeploy Cadent Operative Specific SAV Control Criteria in addition to Existing General Estate SAV Control Criteria.

\subsection{Understanding existing (pre HyDeploy) operative general estate SAV control criteria}

The Keele Estate is an open access site, this means that SAV comes with additional challenges over those expected to be encountered on a closed (gated) site such as defence facility or office block.

The general (existing) SAV Control Criteria are based upon an approved contractor scheme, meaning that baseline health and safety training checks as well as, finance, insurances and other checks are made. The basic credentials required to be met by an operative (employed or contracted) in order to gain access to the site are largely undertaken at an operational level by the contractor, with much of the processing ordinarily handled by the appropriate qualified person on behalf of the contractor (usually not the operative). These credentials are classified as Stage One existing Keele Estate General Site Access Verification Control Criteria and the data pertaining to the completion of such are attributes of the contractor organisation (or the department in the case of an estate employee). In order to meet the SAV control criteria for stage one the contractor operative presenting for work will satisfy the following criteria:

- Employed by an approved Keele contractor,

- Approved Risk Assessments and Method Statements (RAMS) assigned to the job, 
- Approved work place safety plan will have been completed, and

- Approved applications for any and all required permits to work.

Assuming the stage one Existing General Estate SAV Control contractor (or estate employee) attributable criteria are verified as having been met, the operative is now ready to present for site access approval (stage two) at the university estates reception. It is exclusively the responsibility of the operative as an employee of a verified contractor (or employee of the estate), to have completed the Keele Estates Induction and Knowledge Check pertaining to general estate access prior to being granted access to the site. Data pertaining to the completion of this induction then is an attribute of the operative. The Keele Estates Induction is presented in the form of a training video with audio, followed by a written knowledge check. The Estates Induction is undertaken in the Keele estates building prior to first estate access and is valid for a period of 12 months post completion. Upon expiry of the Estates Induction and prior to being granted further estate access, the operative will be required to refresh the training. Completion records and expiry data are stored by the Keele Estates operations team.

Further to Existing General Estate SAV Control Arrangements there are occasions where site within site (sub sites) do exist, this is most commonly the case when a capital construction project is being undertaken, such as the construction and installation of the on-site electrolyser supplying hydrogen to the gas network for the duration of the HyDeploy project. Where sub sites do exist the principle contractor will take responsibility for the activities (including SAV arrangements) up until the point when the project is handed over to Keele University (i.e when the capital phase on the sub site is completed). Before satisfying SAV Control Arrangements for a sub site, the operative would first need to satisfy Existing General Estate SAV Control Criteria.

\subsection{Understanding first line contractor gas network engineers SAV control arrangements specifically for Keele HyDeploy}

The project consortium recognises the vital importance of public safety. Due to the complex nature of the project meant it was necessary for the consortium lead to seek regulatory exemptions from the UK HSE in the planning stage. Following a six-month review and consultation period in collaboration with all consortium partners including the UK HSL, regulatory exemption was granted by the UK HSE.

As part of the pre project safety review by the HSL, over 200 emergency and scheduled maintenance procedures were assessed, the findings of which resulted in the development of supplementary guidance for first line engineers attending to gas emergencies and meter works at the Keele University estate.

The supplementary guidance produced to support the implementation of the regulatory exemption means that operatives are required to conduct tasks in a way which differs from their regular (regulatory based) job training (known as EM72). As such Cadent and Keele University have worked together to develop training materials which are designed to equip operatives with the necessary supplementary information. This information enables those having completed the course to discharge duties safely in the HyDeploy environment (Keele University). The supplementary information has been compiled into a document called 'EM72Hhdrogen' (EM72H) which forms the basis for a one day training course, with completion of this course being a requirement for all operatives being made available [via the cadent job scheduling system] to attend gas 
emergencies and meter works. The one day training course certificate (also know as a ticket) is valid for 12 months post completion.

For the purposes of the SAV control process, the HyDeploy project is technically classified as a sub site of the estate, with the subsite being accessible only to Cadent employees who have met the HyDeploy Cadent Operative Specific SAV Control Criteria in addition to the General Estate SAV Control Criteria.

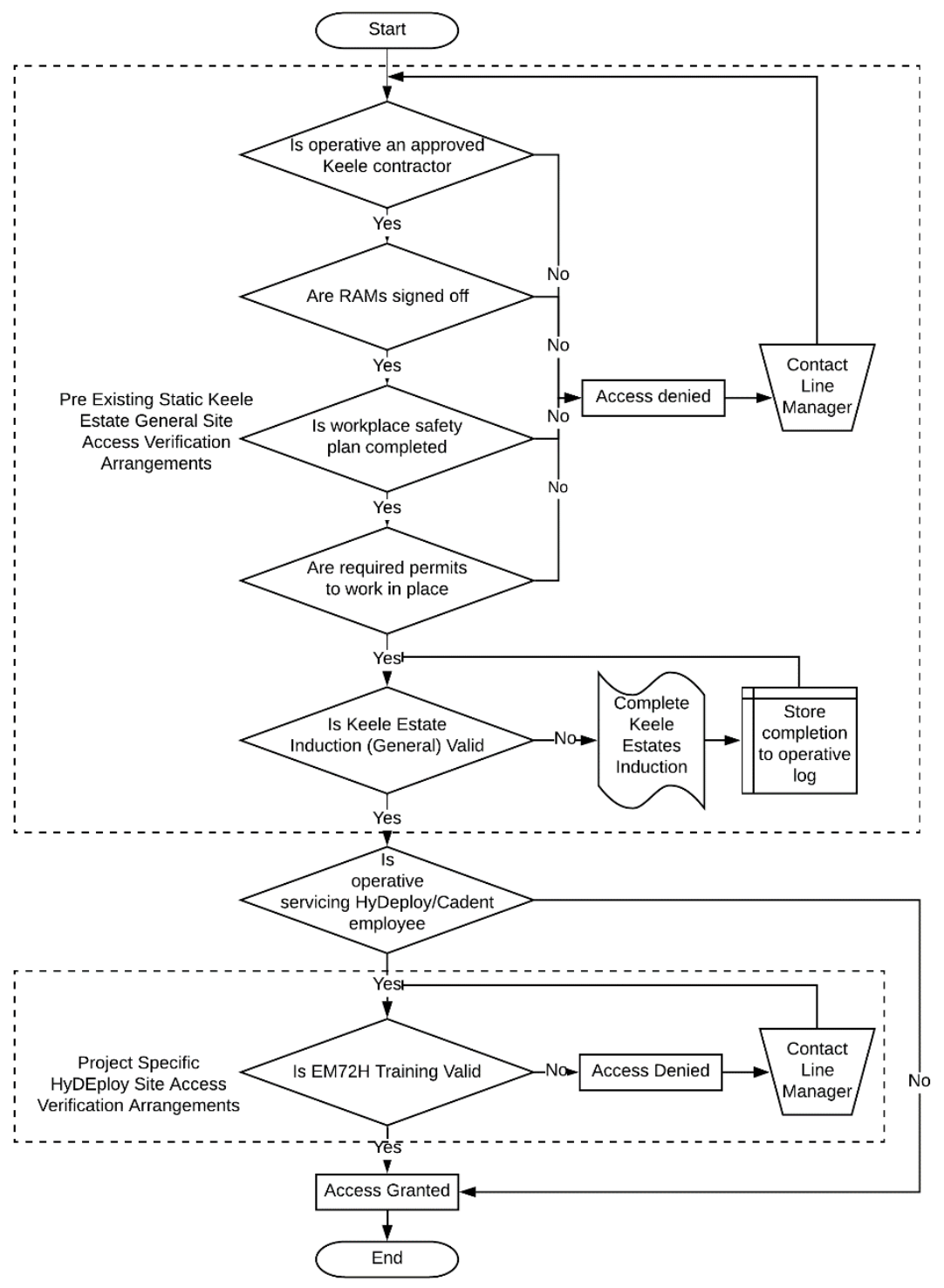

Fig. 1. Existing HyDeploy Cadent operative specific SAV control criteria

\section{RESULTS ANALYSIS}

Having reviewed and agreed the Existing HyDeploy Cadent Operative Specific SAV Control Criteria both research participants completed the initial questionnaire. The open ended nature of the questionnaires required a qualitative content approach to analyse the results. A number of key themes were able to be identified from the analysis of the results. 
Change management was identified as the key theme with emerging smart technologies roll outs such as HyDeploy. Both participants reported that change management in respect of safety procedures (and associated health and safety training) requires very careful planning to ensure that compliance with the regulatory exemptions is maintained, and critically that the specifics of the exemption are understood by all operatives.

Both participants identified that the existing methods of SAV control do ensure that only trained operatives are authorised to attend site when emergencies are reported. Equally though both acknowledged that there are opportunities to further leverage technology to improve the solution. Most notably both participants identified operational challenges (specifically cost) regarding refresher training (post ticket expiry) as being key concern with one participant commenting, "renewal of authorisation cards and any ongoing changes that require an update or change of procedure within EM72H need to be disseminated and verified and at the moment the only process to complete this is by [face to face] training sessions/toolbox talks and Keele site visits. An alternative cost effective solution to change management and authorisation would be beneficial." Indeed "Due to the widespread layout of gas operatives it would be unpractical and expensive to get all the operatives to site." The compliance threshold for change management such as that required for this project requires very specific SAV control arrangements to be put in place, evidently there are cost implications arising from the operational challenges pertaining to the implementation of such control arrangements.

The second stage of the research was designed to determine research participant apparent acceptance of a solution to improve the current SAV arrangements (Figure 2). The solution being researched presents both the Estate owner/operator (Keele Uniersity) and the Contractor company (Cadent) the opportunity to improve the quality, accessibility and availability relevant information in a way which supports the communication of information which is of specific relevance to the project.

The SAV process can be developed to include new additional micro credentials which are able to cover a variety of topics. One such example of an additional credential can be seen in the form of 'procedural amendment content'. In the case of a procedural amendment, under the existing arrangements an email would typically be sent out to the operative group, with all amendments being highlighted. The proposed arrangements supported by the solution being researched would enable content to be pushed to the user group (in this case all 100 operatives), providing the operative with a notification to the effect that new content is now required to be completed prior to the next operative visit to Keel University.

Note scheduling of content completion can be set according to the specific objectives of the content roll out, example immediate completion required. Prior to arrival at site the operative would access the content typically presented in the form of a short video, with completion recorded back to the system to support the SAV verification process and to support administration and auditing processes on both the estate owner/operator and client contractor side. Additional content can be pushed out by the solution, with short assessments set as content completion criteria where deemed relevant.

Figure 2 (Proposed Site Access Verification (SAV) Control Criteria Flow is representative of the content types which can be pushed out and the objectives which are able to be met by completion of such content. Additional content types covered by the solution include, reactive safety content which may have been generated according to the outcome of an accident investigation or near miss. The flow diagram was shared 
with the research participants for review prior to the sharing and subsequent completion of the final stage questionnaire.

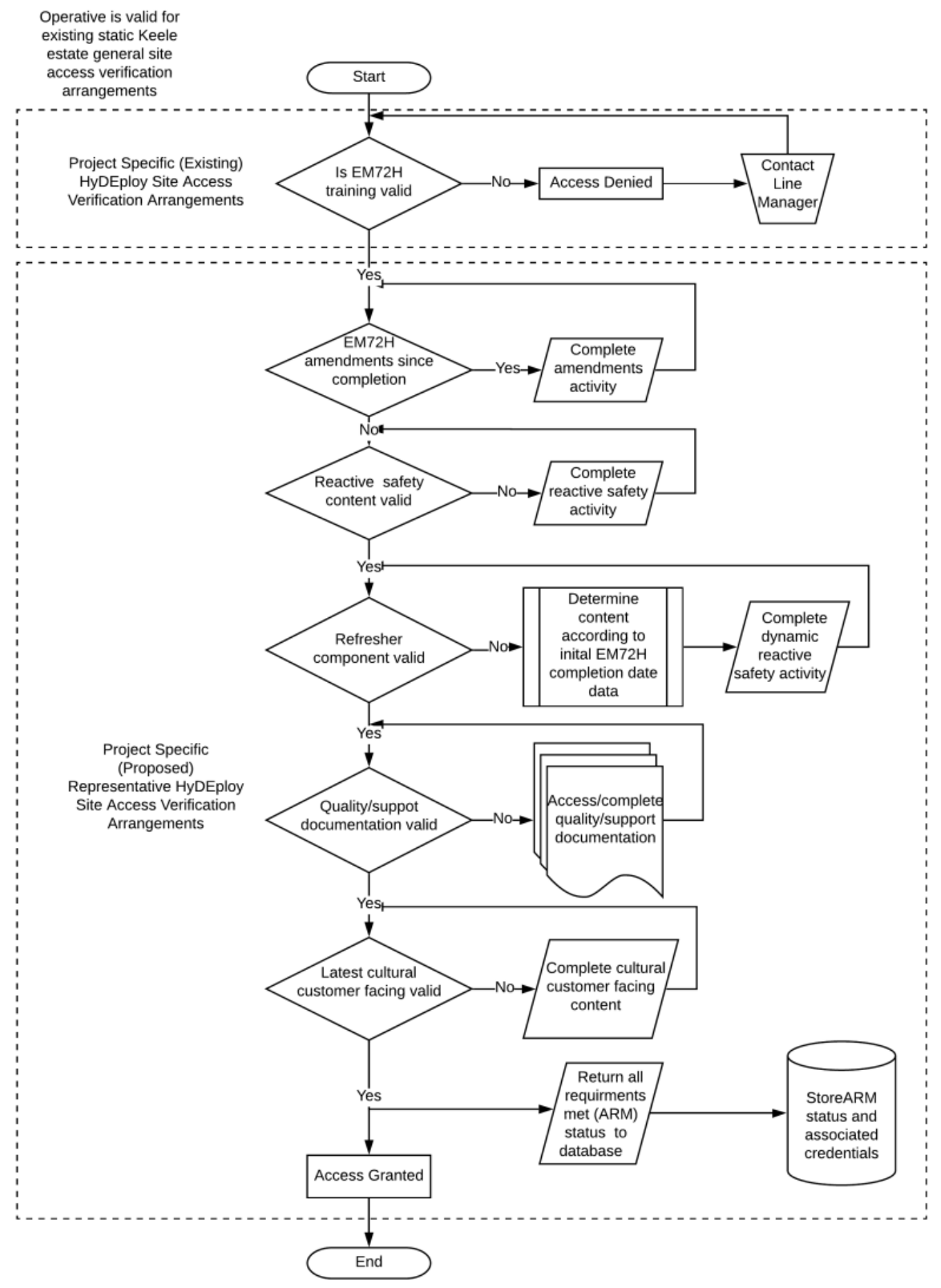

Fig. 2. Proposed Site Access Verification (SAV) Control Criteria Flow

Both participants agreed that the proposed solution would improve the level of confidence in the compliance position regarding HyDeploy SAV arrangements, with one research participant noting that the proposed arrangements would "enable a management team to have confidence that knowledge is transferred in a timely manner" and that data made available via knowledge check reporting would enable management teams to "determine the effectiveness of the training medium". A key theme emerging from the analysis of data from this stage was the perceived ability of the proposed solution to efficiently support the communication of minor alterations to existing procedures which is especially useful in pathfinder projects such as this one where "[site] specific hazards" exist. 
When asked about potential challenges that may affect the implementation of such a solution, staff engagement and the need to ensure staff do not experience a feeling of "alienation" from the face to face training process was highlighted as a concern. Additional concerns raised related to, adequate process documentation and maintenance, the provision of available hardware, and the fact that e-learning can be a "frustrating" experience.

Regarding potential additional benefits of the proposed solution one research participant identified the opportunity to create communities around projects where specific risks exist, thereby leveraging the technology to ensure that opportunities which exist in classroom situations to "promote open conversations" are not missed and continue to inform training outcomes and development.

\section{CONCLUSIONS}

The HyDeploy project is demonstrative of the type of risk scenarios which may reasonably be expected to exist as the range of smart technologies solutions grows as part of a global response to the challenges associated with a growing population, increasing urbanisation and global warming. To address the increasing risk profile associated with this transition, specific responses are required to ensure that operatives are familiar with the change process and are protected from the increased risk profile.

This research demonstrated the specific SAV arrangements developed in collaboration with the UK HSL and implemented at the Keele University site. The research found that while the existing arrangements were required to be implemented to account for regulatory exemptions and ensure that associated changes to existing procedures and protocols relating to the presence of hydrogen (blended with natural gas) are communicated to a contractor group of 100 persons. The arrangements ensure that only trained authorised engineers attend the estate when emergencies are reported.

Expert input found that the current arrangements face change management challenges and are not cost effective especially in situations where amendments to completed training are needed. The research proposed a technology-enabled SAV solution as a way for improving the training outcomes for operatives and strengthening the confidence in the compliance position of contractors. Experts perceived the solution as having clear potential to improve the perceived compliance position of both the estate owner and the contract provider, asserting that operatives would be more well informed of change process relating to project specific risks under the proposed arrangements.

\section{REFERENCES}

Ahmed, F., Ahmed, N., Heitmueller, A., Gray, M., Atun, R. (2017). Smart cities: Health and safety for all. The Lancet Public Health 2, 9.

Aik, C.T. and Tway, D.C. (2006) Elements and principles of training as a performance improvement solution. Performance Improvement, 45(3), 28-32.

Blocker, M. J. (2005) E-learning: an organizational necessity, White Paper. Available at: 5www. rxfrohumanperformance.com4 (accessed 31 January 2006).

Carbonari, G., Stravoravdis, S., Gausden, C. (2018). Improving FM task efficiency through BIM: A proposal for BIM implementation. Journal of Corporate Real Estate, 20(1), 4-15. 
Ertmer, P. A., Newby, T. J. (2013). Behaviourism, cognitivist, constructivism: Comparing critical features from an instructional design perspective. Performance Improvement Quarterly, 26(2), 43-71.

Gao, Y., Gonzalez, V., Yiu, T. (2019). The effectiveness of traditional tools and computeraided technologies for health and safety training in the construction sector: A systematic review. Computers \& Education, 138, 101-115.

Harvey, D. (2018). MAKE WAY FOR MICRO-CREDENTIALS. Canadian HR Reporter, 31(3), 19.

Heaton, J., Parlikad, A. (2019). A conceptual framework for the alignment of infrastructure assets to citizen requirements within a Smart Cities framework. Cities, 90, 32-41.

Ho, C., Dzeng, R. (2010). Construction safety training via e-Learning: Learning effectiveness and user satisfaction. Computers \& Education, 55(2), 858-867

Hilal, M., Maqsood, T., Abdekhodaee, A. (2019). A hybrid conceptual model for BIM in FM. Construction Innovation, 19(4), 531-549.

Kassem M., Benomran L., Teizer J. (20170) Virtual environments for safety learning in construction and engineering: seeking evidence and identifying gaps for future research, Visualization in Engineering, Springer, 5:16.

Öberg, C., Graham, G., Hennelly, P. (2017). Smart cities. IMP Journal, 11(3), 468-484

Partnerships for SDGs. United smart cities. (2016). https://sustainabledevelopment.un.org/partnership/?p=10009 [Accessed 02 Jan. 2020].

Rapid urbanisation. https://www.pwc.co.uk/issues/megatrends/rapid-urbanisation.html [Accessed 02 Jan. 2020].

United Nations Population Growth: https://www.un.org/en/sections/issuesdepth/population/index.html. [Accessed 02 Jan. 2020].

United Nations Revision of World Urbanisation Prospects: https://www.un.org/development/desa/publications/2018-revision-of-worldurbanization-prospects.html. [Accessed 02 Jan. 2020].

Shamsudin, N.Md. (2018). Virtual Reality for Construction Occupational Safety and Health Training: A Review. Advanced Science Letters, 24(4), 2444-2446.

Webb, H. W., Gill, G., Poe, G. (2005). Teaching with the case method online: Pure versus hybrid approaches. Decision Sciences Journal of Innovative Education, 3(2), 223-250.

Wetzel, E.M., Thabet, W. Y. (2018). A case study towards transferring relevant safety information for facilities maintenance using BIM. Journal of Information Technology in Construction (ITcon), 23, 53-74.

Williams, Q., Ochsner, M., Marshall, E., Kimmel, L., Martino, C. (2010). The impact of a peer-led participatory health and safety training program for Latino day labourers in construction. Journal of Safety Research, 41(3), 253 -261. 